\title{
Electricity Market Stochastic Dynamic Model and Its Mean Stability Analysis
}

\author{
Zhanhui Lu, ${ }^{1}$ Weijuan Wang, ${ }^{1}$ Gengyin $L i,{ }^{2}$ and Di Xie ${ }^{1}$ \\ ${ }^{1}$ School of Mathematics and Physical Science, North China Electric Power University, Beijing 102206, China \\ ${ }^{2}$ School of Electrical and Electronic Engineering, North China Electric Power University, Beijing 102206, China \\ Correspondence should be addressed to Zhanhui Lu; luzhanhui@ncepu.edu.cn
}

Received 7 July 2014; Accepted 10 September 2014; Published 5 November 2014

Academic Editor: Weihai Zhang

Copyright (C) 2014 Zhanhui Lu et al. This is an open access article distributed under the Creative Commons Attribution License, which permits unrestricted use, distribution, and reproduction in any medium, provided the original work is properly cited.

\begin{abstract}
Based on the deterministic dynamic model of electricity market proposed by Alvarado, a stochastic electricity market model, considering the random nature of demand sides, is presented in this paper on the assumption that generator cost function and consumer utility function are quadratic functions. The stochastic electricity market model is a generalization of the deterministic dynamic model. Using the theory of stochastic differential equations, stochastic process theory, and eigenvalue techniques, the determining conditions of the mean stability for this electricity market model under small Gauss type random excitation are provided and testified theoretically. That is, if the demand elasticity of suppliers is nonnegative and the demand elasticity of consumers is negative, then the stochastic electricity market model is mean stable. It implies that the stability can be judged directly by initial data without any computation. Taking deterministic electricity market data combined with small Gauss type random excitation as numerical samples to interpret random phenomena from a statistical perspective, the results indicate the conclusions above are correct, valid, and practical.
\end{abstract}

\section{Introduction}

As we all know, stochastic differential equation has been booming as a cross-discipline of probability theory and differential equation. Based on the stability theory of deterministic differential equations and stochastic process theory, the stability theory of stochastic differential equation gets rapid development and has quite widespread applications. Ranging from a specific control system to a social system, a financial system, or an ecosystem, whether the system is large or small, it always runs in random or persistent disturbance. With the random disturbance, it is essential that the system maintains a predetermined running or operating state, instead of wavering or being out of control. The socalled stability for stochastic system movement is aimed at researching the stability of its equilibrium state from the perspective of statistics. That is to say, the disturbed movement, deviating from the equilibrium state, can return to the equilibrium state or restrict to its finite neighborhood when it only relies on the internal structure factors of the system.
Electricity market is based on power system and electricity market is the operating mode of power system at the same time. The economic stability of electricity market and the physical stability of power system are linked and affect each other. In 1990, Beavis and Dobbs began to study economic system stability [1]. For twenty years now, the research on the dynamic evolution and stable behavior of economy and management system has been arousing wide attention in the fields of mathematical economics, system science, and so forth. In particular, the stability analysis of economic systems branch is the emphasis and difficulty in this field and is of great significance as well. While the research of electricity market stability begins to perk up in recent years [2-11], market mechanism is employed to reasonably allocate power resources in electricity market. The research on electricity market stability is very significant for regulating the market supply and demand. Combining the market dynamics, the electricity market dynamic model is proposed by Alvarado [2]. After cooperation with Meng and others, Alvarado created a new market model considering 
the electrical energy unbalanced factors as well as the power system's dynamic factors in the dynamic market model [35]. For all of the above market dynamics models, numerical eigenvalue method was applied to study the stability of electricity market. An electricity market dynamic model proposed by Alvarado is generalized and the effect of market clearing time and price signal delay on system stability is analyzed in [12]. With the dynamic market model, the role that futures markets may have on the clearing prices and on altering the volatility and potential instability of real time prices and generator output is examined by Watts and Alvarado [13]. An appropriate modeling of system controllers is provided in [14] to account for the effects of power system controllers and stability on power dispatch and electricity market prices. For a kind of dynamic power market models with congestion, a series of sufficient conditions are provided to determine the stability of power market in [15]. Combining the market dynamic model put forward by Alvarado, the stability of electricity market is theoretically analyzed and the determine conditions of stability on electricity market [16]. Actually, the involved discussion about stability above is derived from Lyapunov asymptotical stability of equilibrium in the deterministic differential equation theory.

With the integration of renewable power and electric vehicle, the power system stability is of growing concern because the active power generated by the renewable energy and absorbed by the electric vehicle varies randomly. A framework is presented in [17] to study the impact of stochastic active/reactive power injections on power system dynamics with a focus on time scales involving electromechanical phenomena. In order to investigate the small signal stability of the single machine infinite bus power system, the stochastic stability analysis method [18] which is applied based on Lyapunov stability and stochastic stability theory is introduced. A new method [19] integrating the transient energy function and recloser probability distribution functions is provided to analyze structure-preserved power system transient stability. Based on the deterministic power system model, the power system stochastic model under small Gauss type random excitation is provided and the mean stability and mean square stability of the power system model are verified theoretically in [20]. The research on stochastic stability of power system has been increasing recently [1720]. However, the stochastic differential equation stability theory on electricity market investigation is rarely applied.

Based on the electricity market dynamic model proposed by Alvarado, a stochastic electricity market dynamic model is presented under the assumption that the generator cost function and consumer utility function are the quadratic function in this paper. The electricity market model proposed by Alvarado can be seen as a special case of the stochastic electricity market dynamic model. The small Gauss type random excitation is provided to describe the random nature of demand sides. Using the theory of stochastic differential equations, stochastic process theory, and eigenvalue techniques, the stochastic electricity market model is analyzed theoretically and the determining conditions of the mean stability are given as follows. If the demand elasticity of suppliers is positive and the demand elasticity of consumers is negative or the demand elasticity of suppliers is nonnegative and the demand elasticity of consumers is negative, then the electricity market dynamic model under Gauss type of random small excitation is of mean stability. That is to say, we can judge its mean stability directly by the initial data without any numerical computation. Finally, taking deterministic electricity market data combined with small Gauss type random excitation as numerical samples, from a statistical perspective to examine the numerical results, it is demonstrated that the obtained conclusions of stability for the stochastic electricity market model are not only correct and effective, but also practical and concise.

\section{The Stochastic Dynamic Modeling of Electricity Market}

2.1. Alvarado Electricity Market Model. Let generator cost functions and consumer utility functions be quadratic functions. If a supplier observes a market price $\lambda$, above its production cost $\lambda_{g i}$, it is assumed that the supplier will expand production until the marginal cost of production equals the price. The rate of expansion is proportional to the difference between the observed price and the actual production cost. The speed with which the generation power output $P_{g i}$ of supplier $i$ can respond is supplier-dependent, which is denoted by a time constant $\tau_{g i}$ for supplier $i$. The above yields the following differential equation to describe the dynamic behavior of electricity market [2]:

$$
\tau_{g i} \dot{P}_{g i}=\lambda-b_{g i}-c_{g i} P_{g i} \quad(i=1,2, \ldots, m),
$$

where $P_{g i}$ is the generation power output, the speed of power output is denoted by a time constant $\tau_{g i}, \lambda$ is the price at any given time, $b_{g i}+c_{g i} P_{g i}$ is the marginal cost of supplier $i, c_{g i}$ is the demand elasticity of supplier $i$, and $b_{g i}$ is the linear cost coefficient of supplier $i$.

As for consumers, the equation describing a consumer behavior is

$$
\tau_{d j} \dot{P}_{d j}=\lambda+b_{d j}+c_{d j} P_{d j} \quad(j=1,2, \ldots, n),
$$

where $P_{d j}$ is a consumer's demand, $\tau_{d j}$ is the expansion speed of consumption demand, $b_{d j}+c_{d j} P_{d j}$ is the marginal benefit of consumer $j, c_{d j}$ is the demand elasticity of consumer $j$, and $b_{d j}$ is the linear cost coefficient of consumer $j$. In addition, $P_{d j}$ and $P_{g i}$ satisfy

$$
\sum_{i=1}^{m} P_{g i}=\sum_{j=1}^{n} P_{d j}
$$

Considering the congestion of power market, using flow distribution factors, a single congested condition can be represented as a scalar additional equality constraint:

$$
\begin{aligned}
s_{g 1} P_{g 1} & +s_{g 2} P_{g 2}+\cdots+s_{g m} P_{g m} \\
& +s_{d 1} P_{d 1}+s_{d 2} P_{d 2}+\cdots+s_{d n} P_{d n}=s_{1} .
\end{aligned}
$$


Generally, with $n_{s}$ congestion conditions, the complete model of $m$-supplier and $n$-consumer case is

$$
\left[\begin{array}{ll}
T & 0 \\
0 & 0
\end{array}\right]\left[\begin{array}{c}
\dot{\widetilde{P}} \\
\dot{\Lambda}
\end{array}\right]=\left[\begin{array}{cc}
C & S^{T} \\
S & 0
\end{array}\right]\left[\begin{array}{l}
\widetilde{P} \\
\widetilde{\Lambda}
\end{array}\right]+\left[\begin{array}{l}
b \\
s
\end{array}\right],
$$

where $T=\operatorname{diag}\left\{\tau_{g 1}, \tau_{g 2}, \ldots, \tau_{g m}, \tau_{d 1}, \tau_{d 2}, \ldots, \tau_{d n}\right\}, \tau_{g i}>0$, $\tau_{d i}>0, C=\operatorname{diag}\left\{-c_{g 1},-c_{g 2}, \ldots,-c_{g n}, c_{d 1}, c_{d 2}, \ldots, c_{d n}\right\}, \widetilde{P} \in$ $R^{m+n}, \widetilde{\Lambda} \in R^{n_{s}+1}, \widetilde{\Lambda}=\left[\begin{array}{llll}\lambda & \mu_{1} & \cdots & \mu_{n_{s}}\end{array}\right]$, and $\mu_{k}$ is the Lagrange multiplier for $k=1,2, \ldots, n_{s}$. The matrix $S$ corresponds to the sensitivities of the constraints

$$
\left[\begin{array}{cccccc}
1 & \cdots & 1 & -1 & \cdots & -1 \\
s_{11} & \cdots & s_{1 m} & s_{1 m+1} & \cdots & s_{1 m+n} \\
\vdots & \cdots & \vdots & \vdots & \vdots & \vdots \\
s_{n_{s} 1} & \cdots & s_{n_{s} m} & s_{n_{s} m+1} & \cdots & s_{n_{s} m+n}
\end{array}\right],
$$

where the first line represents the power balance conditions; $b=\left[\begin{array}{llllllll}-b_{g 1} & -b_{g 2} & \cdots & -b_{g m} & b_{d 1} & b_{d 2} & \cdots & b_{d n}\end{array}\right]^{\mathrm{T}}$ is a cost vector of linear coefficients; $s=\left[\begin{array}{lllll}0 & s_{1} & s_{2} & \cdots & s_{n_{s}}\end{array}\right]^{\mathrm{T}}$ is a vector with the value of the fixed demand, where $s_{i}$ is the value of the right-hand side for $i=1,2, \ldots, n_{s}$ in the constraint equations in the remaining positions.

2.2. Electricity Market Stochastic Model. The system (5) has at least one equilibrium point. Through transformation, it could be changed as follows:

$$
\left[\begin{array}{ll}
T & 0 \\
0 & 0
\end{array}\right]\left[\begin{array}{c}
\dot{P} \\
\dot{\Lambda}
\end{array}\right]=\left[\begin{array}{cc}
C & S^{T} \\
S & 0
\end{array}\right]\left[\begin{array}{l}
P \\
\Lambda
\end{array}\right] .
$$

Usually, there exists $m+n>n_{s}+1$. Let $\operatorname{rank}(S)=n_{s}+1$ and let $S=\left(S_{1}, S_{2}\right)$, where $S_{1}$ corresponds to a nonsingular $\left(n_{s}+1\right) \times\left(n_{s}+1\right)$-order submatrix of $S$. The matrices $T$ and $C$ can be divided into the following form: $T=\operatorname{diag}\left\{T_{1}, T_{2}\right\}$ and $C=\operatorname{diag}\left\{C_{1}, C_{2}\right\}$, where $T_{1}$ and $C_{1}$ are $\left(n_{s}+1\right) \times\left(n_{s}+1\right)$ order diagonal matrices; $T_{2}$ and $C_{2}$ are $q \times q$-order diagonal matrices, and $q=(m+n)-\left(n_{s}+1\right)$. So $(7)$ can be formed as

$$
\left[\begin{array}{ccc}
T_{1} & 0 & 0 \\
0 & T_{2} & 0 \\
0 & 0 & 0
\end{array}\right]\left[\begin{array}{c}
\dot{P}_{1} \\
\dot{P}_{2} \\
\dot{\Lambda}
\end{array}\right]=\left[\begin{array}{ccc}
C_{1} & 0 & S_{1}^{\mathrm{T}} \\
0 & C_{2} & S_{2}^{\mathrm{T}} \\
S_{1} & S_{2} & 0
\end{array}\right]\left[\begin{array}{c}
P_{1} \\
P_{2} \\
\Lambda
\end{array}\right] .
$$

Since the matrix $S_{1}$ is nonsingular, reduction and elimination to $P_{1}$ yield the following purely reduced differential equation: $-T_{1} S_{1}^{-1} S_{2} \dot{P}_{2}=-C_{1} S_{1}^{-1} S_{2} P_{2}+S_{1}^{\mathrm{T}} \Lambda$. By obtaining $\Lambda$ from the above and substituting it into the second group equation of (8), we have

$$
\left(T_{2}+S_{3}^{\mathrm{T}} T_{1} S_{3}\right) \dot{P}_{2}=\left(C_{2}+S_{3}^{\mathrm{T}} C_{1} S_{3}\right) P_{2} .
$$

Put $S_{3}=S_{1}^{-1} S_{2}, T_{3}=T_{2}+S_{3}^{\mathrm{T}} T_{1} S_{3}$, and $C_{3}=C_{2}+S_{3}^{\mathrm{T}} C_{1} S_{3}$; the system (9) could be formed as follows:

$$
T_{3} d P_{2}=C_{3} P_{2} d t
$$

Considering the random nature of demand sides, after adding a random excitation term to the right-hand side of
(10), the electricity market stochastic model can be described as

$$
T_{3} d P_{2}(t)=C_{3} P_{2}(t)+Q d B(t) \quad t \in[0,+\infty),
$$

where the initial value $P_{2}(0)=P_{0}$ is bounded. $B(t)$ is the $n$ dimensional Wiener process. $P_{0}$ and $B(t)$ are independent of each other. $P_{2}(t)$ and $Q$ are $n$-dimensional column vectors.

\section{Stochastic Differential Equation Theories}

In order to give the determining conditions of stability for the electricity market stochastic model (11), we should introduce some theory referring to stochastic differential equation firstly. For details, see literature [21].

\subsection{Stochastic Differential Equation}

Definition 1 (see [21]). Vector stochastic differential equation with Gauss type white noise can be written as

$$
d X(t)=f(X(t), t) d t+G(X(t), t) d B(t),
$$

where $X(t)=\left(X_{1}(t), X_{2}(t), \ldots, X_{n}(t)\right)^{\mathrm{T}}$ is the $n$-dimensional vector random variable; $B(t)=\left(B_{1}(t), B_{2}(t), \ldots, B_{n}(t)\right)^{\mathrm{T}}$ is the $n$-dimensional Wiener process. The initial value $X\left(t_{0}\right)=$ $X_{0}$ is bounded. $X_{0}$ and $B(t)$ are independent of each other. The derivative form of $B(t)$ is denoted as $d B(t) / d t=W(t)$ for $t \in\left[t_{0}, T\right]$, where $W(t)$ is the vector Gaussian white noise. If $f(X(t))$ and $G(X(t))$ are the linear functions of $X(t)$, namely, $f(X(t), t)=A(t) X(t)+a(t)$, the special linear stochastic differential equation can be obtained as follows:

$$
d X(t)=(A(t) X(t)+a(t)) d t+Q(t) d B(t),
$$

where $a(t)$ is the $n$-dimensional vector, $A(t)$ is an $n \times n$-order matrix, and $Q(t)$ is an $n \times m$-order matrix. If $A(t)=A$ and the special linear stochastic differential equation (13) satisfies measurability, Lipschitz continuity, linear growth conditions, and initial condition [21], then its solution process can be expressed as

$$
X(t)=e^{A\left(t-t_{0}\right)} X_{0}+\int_{t_{0}}^{t} e^{A(t-s)}(a(s) d s+Q(s) d B(s)) .
$$

Particularly, if $a(t)=0$ and $Q(t)=Q$, its solution process can be written as

$$
X(t)=e^{A\left(t-t_{0}\right)} X_{0}+\int_{t_{0}}^{t} e^{A(t-s)} Q d B(s) .
$$

Let $\|\cdot\|_{2}$ denote norm; it refers to modular arithmetic and 2-norm for vector and matrix, respectively.

Definition 2 (see [22]). If the solution process $X(t)$ of the stochastic differential equation (12) satisfies

$$
\lim _{t \rightarrow \infty} E\|X(t)\|_{2}<c,
$$

where $c$ is constant and greater than zero, then the system is of mean stability.

Mean stability implies that the mean value of the response of the system with random input is bounded. 


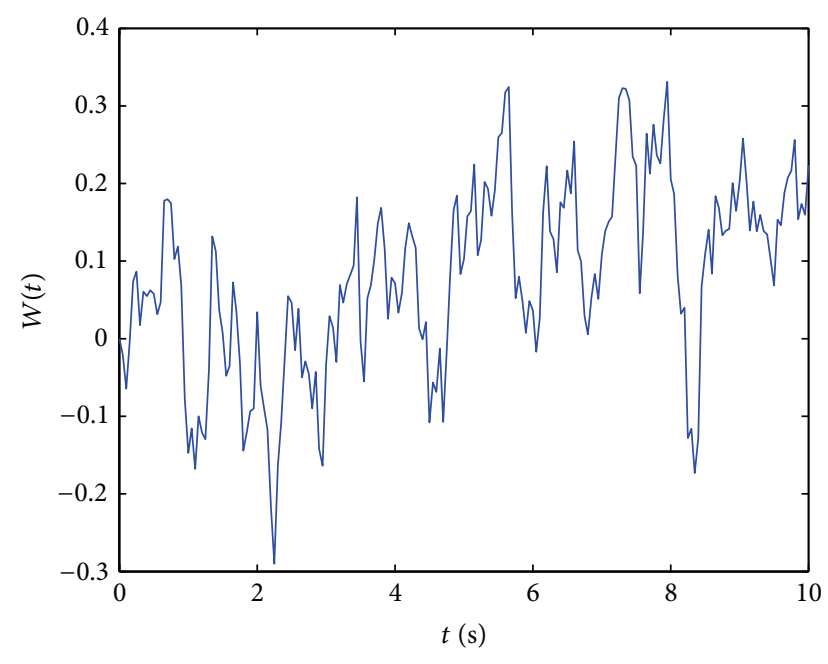

FIGURE 1: Simulation path of Brown motion.

3.2. Numerical Computing Method. Generally, it is difficult to obtain the analytical solution of stochastic differential equation, while it is feasible to get the trajectory of solution process with numerical computing methods to approach the exact solution. Euler-Maruyama (EM) numerical method is one of important numerical methods for the solution of stochastic differential equation [23]. Let the stochastic process of $X(t)$ be the process of solving (12). Difference iterative format of EM numerical method is as follows:

$$
X\left(t_{j+1}\right)=X\left(t_{j}\right)+f\left(X\left(t_{j}\right), t_{j}\right) \Delta t+G\left(X\left(t_{j}\right), t_{j}\right) \Delta W_{j},
$$

where $\Delta t=\left(T-t_{0}\right) / N, N$ is a certain positive integer, and $t_{j}=t_{0}+j \Delta t, j=0,1,2, \ldots, N$. Based on the conditions [21] satisfied by Wiener process, we have $\Delta W_{j}=W\left(t_{j+1}\right)-$ $W\left(t_{j}\right)$ and $\Delta W_{j} \sim \sqrt{\Delta t} N(0,1)$, where $W\left(t_{j}\right)$ is the discrete Wiener process. A simulation path of Wiener process can be produced on the interval $[0,10]$, such as $N=200$, and it can be seen that the Wiener process is continuous while it is not differentiable in Figure 1.

The contents above are cited from the related literature. The following sections are the contribution of the paper, in which the stability problems and the numerical examples of random response are investigated.

\section{Mean Stability of Electricity Market Stochastic Model}

In this section, the determining conditions of mean stability for the electricity market stochastic model (11) will be shown and proved theoretically.

Lemma 3 (see [16]). Let $A_{n \times n}$ be a real positive semidefinite matrix, and let $S_{n \times r}$ be a certain real matrix (as long as $S^{\mathrm{T}} A S$ is meaningful); then, $B=S^{\mathrm{T}} A S$ is a positive semidefinite matrix.

Lemma 4 (see [16]). The sum of positive definite matrix and positive semidefinite matrix will be positive definite matrix; the sum of negative definite matrix and negative semidefinite matrix will be negative definite matrix; the sum of two positive definite matrix will be positive definite matrix; the sum of two negative definite matrix will be negative definite matrix.

Lemma 5 (see [20]). If $A$ is an $n \times n$ matrix and $\lambda_{1}, \lambda_{2}, \ldots, \lambda_{n}$ are the eigenvalues of $A$, then there is a constant $L>0$ satisfying

$$
\left\|e^{A}\right\|_{2} \leq L e^{\lambda}
$$

where $\lambda=\max \left\{\operatorname{Re}\left(\lambda_{1}\right), \ldots, \operatorname{Re}\left(\lambda_{n}\right)\right\}$.

Theorem 6. If all the eigenvalues of $C_{3}$ are negative in the electricity market stochastic model (11), then the model is of mean stability.

Proof. Since $T=\operatorname{diag}\left(\tau_{g 1}, \ldots, \tau_{g m}, \tau_{d 1}, \ldots, \tau_{d n}\right)=\operatorname{diag}\left(T_{1}\right.$, $\left.T_{2}\right), T_{1}=\operatorname{diag}\left(\tau_{g 1}, \ldots, \tau_{g m}\right)$, and $T_{2}=\operatorname{diag}\left(\tau_{d 1}, \ldots, \tau_{d n}\right), \tau_{g i}>$ $0, \tau_{d j}>0$, the matrices $T, T_{1}$, and $T_{2}$ are positive definite. By Lemma 3, the matrix $S_{3}^{\mathrm{T}} T_{3} S_{3}$ is positive semidefinite. Then, by Lemma $4, T_{3}=T_{2}+S_{3}^{\mathrm{T}} T_{1} S_{3}$ is a positive definite matrix.

According to literature [16], there exists an $n$-order reversible matrix $T_{33}$ such that $T_{3}=\left(T_{33}^{-1}\right)^{\mathrm{T}} T_{33}^{-1}$. Substituting it into (11) yields that $\left(T_{33}^{-1}\right)^{\mathrm{T}} T_{33}^{-1} d P_{2}(t)=C_{3} P_{2}(t)+Q d B(t)$. By $\left(T_{33}^{-1}\right)^{\mathrm{T}}=\left(T_{33}^{\mathrm{T}}\right)^{-1}$, we have

$$
T_{33}^{-1} d P_{2}(t)=T_{33}^{\mathrm{T}} C_{3} T_{33} T_{33}^{-1} P_{2}(t)+T_{33}^{\mathrm{T}} Q d B(t)
$$

Put $X(t)=T_{33}^{-1} P_{2}(t)$; then, $d X(t)=T_{33}^{-1} d P_{2}(t)$. Equation (19) can be written as

$$
X(t)=T_{33}^{\mathrm{T}} C_{3} T_{33} d X(t)+T_{33}^{\mathrm{T}} \mathrm{Q} d B(t),
$$

where $T_{33} P_{0}=X_{0}$. Its explicit expression is

$$
X(t)=e^{T_{33}^{\mathrm{T}} C_{3} T_{33} t} X_{0}+\int_{0}^{t} e^{T_{33}^{\mathrm{T}} C_{3} T_{33}(t-s)} T_{33}^{\mathrm{T}} Q d B(s) .
$$

The matrix $C_{3}$ is real symmetric so that its eigenvalues are real numbers. On account of the conditions, all the eigenvalues of $C_{3}$ are negative. The matrix $T_{33}^{\mathrm{T}} C_{3} T_{33}$ is the contragradient transformation of $C_{3}$, so they have the same eigenvalues symbol. Let $\lambda_{j}$ denote the eigenvalues of $T_{33}^{\mathrm{T}} C_{3} T_{33}$ for $j=1, \ldots, n$; then, $\lambda_{j}<0$.

By Cauchy-Schwarz inequation, we have

$$
\begin{aligned}
& \left(\mathrm{E}\|X(t)\|_{2}\right)^{2} \\
& \leq \mathrm{E}\|X(t)\|_{2}^{2}=\mathrm{E}\left[X^{\mathrm{T}}(t) X(t)\right] \\
& =\mathrm{E}\left[\left(e^{T_{33}^{\mathrm{T}} C_{3} T_{33} t} X_{0}\right)^{\mathrm{T}} e^{T_{33}^{\mathrm{T}} C_{3} T_{33} t} X_{0}\right] \\
& \quad+\mathrm{E}\left[\left(e^{T_{33}^{\mathrm{T}} C_{3} T_{33} t} X_{0}\right)^{\mathrm{T}} \int_{0}^{t} e^{T_{33}^{\mathrm{T}} C_{3} T_{33}(t-s)} T_{33}^{\mathrm{T}} \mathrm{Q} d B(s)\right]
\end{aligned}
$$




$$
\begin{gathered}
+\mathrm{E}\left[\left(\int_{0}^{t} e^{T_{33}^{\mathrm{T}} C_{3} T_{33}(t-s)} T_{33}^{\mathrm{T}} Q d B(s)\right)^{\mathrm{T}} e^{T_{33}^{\mathrm{T}} C_{3} T_{33} t} X_{0}\right] \\
+\mathrm{E}\left[\left(\int_{0}^{t} e^{T_{33}^{\mathrm{T}} C_{3} T_{33}(t-s)} T_{33}^{\mathrm{T}} Q d B(s)\right)^{\mathrm{T}}\right. \\
\left.\times \int_{0}^{t} e^{T_{33}^{\mathrm{T}} C_{3} T_{33}(t-s)} T_{33}^{\mathrm{T}} Q d B(s)\right] .
\end{gathered}
$$

Using the definition of 2-norm of vector and the expectation properties of nonrandom variable, it can be obtained that

$$
\mathrm{E}\left[\left(e^{T_{33}^{\mathrm{T}} C_{3} T_{33} t} X_{0}\right)^{\mathrm{T}} e^{T_{33}^{\mathrm{T}} C_{3} T_{33} t} X_{0}\right]=\left\|e^{T_{33}^{\mathrm{T}} C_{3} T_{33} t} X_{0}\right\|_{2}^{2}
$$

Because the formal derivative of $B(t)$ is a Wiener process, $\left(e^{T_{33}^{\mathrm{T}} C_{3} T_{33} t} X_{0}\right)^{\mathrm{T}} \int_{0}^{t} e^{T_{33}^{\mathrm{T}} C_{3} T_{33}(t-s)} T_{33}^{\mathrm{T}} \mathrm{Q} d B(s)$ is also a Wiener process and its expectation value of Wiener process is 0 . Hence,

$$
\mathrm{E}\left[\left(e^{T_{33}^{\mathrm{T}} C_{3} T_{33} t} X_{0}\right)^{\mathrm{T}} \int_{0}^{t} e^{T_{33}^{\mathrm{T}} C_{3} T_{33}(t-s)} T_{33}^{\mathrm{T}} \mathrm{Q} d B(s)\right]=0 .
$$

In a similar argument as above, we obtain

$$
\mathrm{E}\left[\left(\int_{0}^{t} e^{T_{33}^{\mathrm{T}} C_{3} T_{33}(t-s)} T_{33}^{\mathrm{T}} Q d B(s)\right)^{\mathrm{T}} e^{T_{33}^{\mathrm{T}} C_{3} T_{33} t} X_{0}\right]=0 .
$$

By the stochastic integral property of Wiener process using real value function, we have [24]

$$
\begin{gathered}
\mathrm{E}\left[\left(\int_{0}^{t} e^{T_{33}^{\mathrm{T}} C_{3} T_{33}(t-s)} T_{33}^{\mathrm{T}} Q d B(s)\right) \int_{0}^{\mathrm{T}} e^{t} e^{T_{33}^{\mathrm{T}} C_{3} T_{33}(t-s)} T_{33}^{\mathrm{T}} \mathrm{Q} d B(s)\right] \\
\quad=\mathrm{E}\left\|\int_{0}^{t} e^{T_{33}^{\mathrm{T}} C_{3} T_{33}(t-s)} T_{33}^{\mathrm{T}} \mathrm{Q} d B(s)\right\|_{2}^{2} \\
\quad=\int_{0}^{t} \mathrm{E}\left\|e^{T_{33}^{\mathrm{T}} C_{3} T_{33}(t-s)} T_{33}^{\mathrm{T}} \mathrm{Q}\right\|_{2}^{2} d s \\
\quad=\int_{0}^{t}\left\|e^{T_{33}^{\mathrm{T}} C_{3} T_{33}(t-s)} T_{33}^{\mathrm{T}} \mathrm{Q}\right\|_{2}^{2} d s .
\end{gathered}
$$

Substituting (23) (26) into (22), it can be yielded as

$$
\begin{aligned}
\mathrm{E} & {\left[X^{\mathrm{T}}(t) X(t)\right] } \\
& =\left\|e^{T_{33}^{\mathrm{T}} C_{3} T_{33} t} X_{0}\right\|_{2}^{2}+\int_{0}^{t}\left\|e^{T_{33}^{\mathrm{T}} C_{3} T_{33}(t-s)} T_{33}^{\mathrm{T}} Q\right\|_{2}^{2} d s .
\end{aligned}
$$

By the norm property and Lemma 5 , the above equation satisfies

$$
\left\|e^{T_{33}^{\mathrm{T}} C_{3} T_{33} t} X_{0}\right\|_{2}^{2} \leq\left\|e^{T_{33}^{\mathrm{T}} C_{3} T_{33} t}\right\|_{2}^{2}\left\|X_{0}\right\|_{2}^{2} \leq m_{0} l_{0}^{2} e^{2 \lambda t}
$$

where $m_{0}=\left\|X_{0}\right\|_{2}^{2}>0, \lambda=\max _{1 \leq j \leq n}\left\{\lambda_{j}\right\}<0$, and $l_{0}$ is a certain positive content. The second item of the right-hand side of above equation satisfies

$$
\begin{aligned}
\int_{0}^{t}\left\|e^{T_{33}^{\mathrm{T}} C_{3} T_{33}(t-s)} T_{33}^{\mathrm{T}} Q\right\|_{2}^{2} d s \\
\quad \leq \int_{0}^{t}\left\|e^{T_{33}^{\mathrm{T}} C_{3} T_{33}(t-s)}\right\|_{2}^{2}\left\|T_{33}^{\mathrm{T}} Q\right\|_{2}^{2} d s \\
\quad \leq m_{1} l_{1}^{2} \int_{0}^{t} e^{2(t-s)} d s=\frac{m_{1} l_{1}^{2}}{2 \lambda}\left(e^{2 \lambda t}-1\right),
\end{aligned}
$$

where $m_{1}=\left\|T_{33}^{\mathrm{T}} \mathrm{Q}\right\|_{2}^{2}>0$.

Therefore, (27) can be changed as

$$
\mathrm{E}\left[X^{\mathrm{T}}(t) X(t)\right] \leq m_{0} l_{0}^{2} e^{2 \lambda t}+\frac{m_{1} l_{1}^{2}}{2 \lambda}\left(e^{2 \lambda t}-1\right) .
$$

According to (22), (30) can be written as

$$
\lim _{t \rightarrow \infty}\left(\mathrm{E}\|X(t)\|_{2}\right)^{2} \leq \lim _{t \rightarrow \infty}\left(m_{0} l_{0}^{2} e^{2 \lambda t}+\frac{m_{1} l_{1}^{2}}{2 \lambda}\left(e^{2 \lambda t}-1\right)\right) .
$$

Owing to $\lambda<0$, we have

$$
\lim _{t \rightarrow \infty}\left(m_{0} l_{0}^{2} e^{2 \lambda t}+\frac{m_{1} l_{1}^{2}}{2 \lambda}\left(e^{2 \lambda t}-1\right)\right)=-\frac{m_{1} l_{1}^{2}}{2 \lambda}>0 .
$$

Hence, if we take a positive constant $L$ that satisfies $L>$ $\sqrt{-m_{1} l_{1}^{2} / 2 \lambda}$ such as $L=\sqrt{-\left(m_{1} l_{1}^{2} / 2 \lambda\right)+1}$, then we get $\lim _{t \rightarrow \infty} \mathrm{E}\|X(t)\|_{2} \leq L$. By Definition 2, the system (11) is of mean stability.

The matrices $C=\operatorname{diag}\left\{C_{1}, C_{2}\right\}, C_{1}=\operatorname{diag}\left\{-c_{g 1}, \ldots\right.$, $\left.-c_{g m}\right\}$, and $C_{2}=\operatorname{diag}\left\{c_{d 1}, \ldots, c_{d n}\right\}$. If the diagonal elements of $C_{1}$ are nonpositive, then the matrix $C_{1}$ is negative semidefinite. By Lemma $3, S_{3}^{\mathrm{T}} C_{1} S_{3}$ is a negative semidefinite matrix. If the diagonal elements of $C_{2}$ are negative, then the matrix $C_{2}$ is negative definite. By Lemma 4 , the matrix $C_{3}=C_{2}+S_{3}^{\mathrm{T}} C_{1} S_{3}$ is real symmetric negative definite. Hence, the following corollary can be obtained.

Corollary 7. If the diagonal elements of $C_{1}$ are nonpositive and the diagonal elements of $C_{2}$ are negative, then the system (11) is of mean stability.

By the theory, it should be worthy to note that if the demand elasticity of suppliers is positive and the demand elasticity of consumers is negative, then the electricity market stochastic model (11) is of mean stability. Furthermore, by the corollary, we can get the conclusion that if the demand elasticity of suppliers is nonnegative and the demand elasticity of consumers is negative, then the electricity market stochastic model (11) is of mean stability.

\section{The Numerical Examples}

Now, we begin to use the theories above to analyze the mean stability of the electricity market stochastic model 


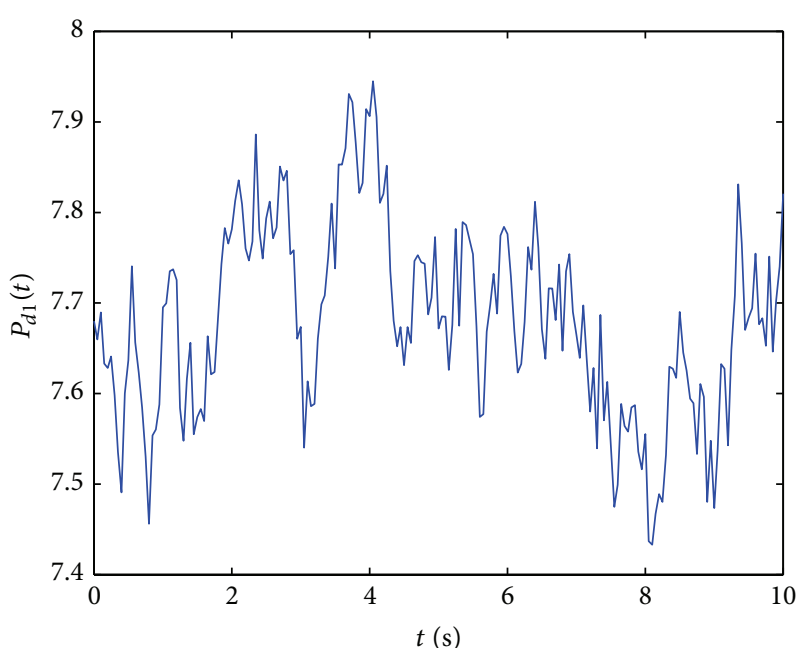

FIGURE 2: The dynamic responses of demand for consumer 1 over a path.

(11) specifically. Some computation results are presented as follows. These deterministic data are derived from Table 4 in [2], corresponding to a determinacy electricity market dynamic model, proposed by Alvarado.

Example 8. In the electricity market stochastic model (11), consider the set of differential/algebraic equations (DAE) corresponding to the case $n_{s}=2$ of three suppliers and two consumers. The demands elasticity of suppliers is $0.3,0.5$, and 0.2 , and response speeds of generation power output are 0.1 , 0.3 , and 0.2 , respectively; the demands elasticity of consumers is -0.5 and -0.6 , and expansion speeds of consumer demands are 0.2 and 0.25 , respectively. The steady state values of the electricity demand are 7.68 and 8.05 , respectively.

When $n_{s}=2$, according to the data above, there are the following in model (11):

$$
\begin{array}{cc}
C_{1}=\operatorname{diag}\{-0.3,-0.5,-0.2\}, & C_{2}=\operatorname{diag}\{-0.5,-0.6\}, \\
T_{1}=\operatorname{diag}\{0.1,0.3,0.2\}, & T_{2}=\operatorname{diag}\{0.2,0.25\}, \\
S_{1}=\left[\begin{array}{ccc}
1 & 1 & 1 \\
0.1 & -0.1 & 0 \\
0.2 & 0 & 0.3
\end{array}\right], & S_{2}=\left[\begin{array}{cc}
-1 & -1 \\
0.1 & -0.1 \\
-0.1 & -0.1
\end{array}\right], \\
P_{2}(t)=\left[\begin{array}{ll}
P_{d 1}(t) & P_{d 2}(t)
\end{array}\right]^{\mathrm{T}} .
\end{array}
$$

Obviously, the matrices $C_{1}$ and $C_{2}$ are negative definite. According to the theorem, it can be concluded that this stochastic dynamic model on electricity market is of mean stability. Combined with the data above, some numerical simulation and computation results are illustrated with the random excitation intensity of $Q=\left[\begin{array}{ll}0.2 & 0.3\end{array}\right]^{\mathrm{T}}$.

Referring to Section 3.2, the simulations of system (11) are performed using EM method and the dynamic responses of $P_{d 2}(t)$ and $P_{d 2}(t)$ solution processes on a path are shown in Figures 2 and 3 , respectively.

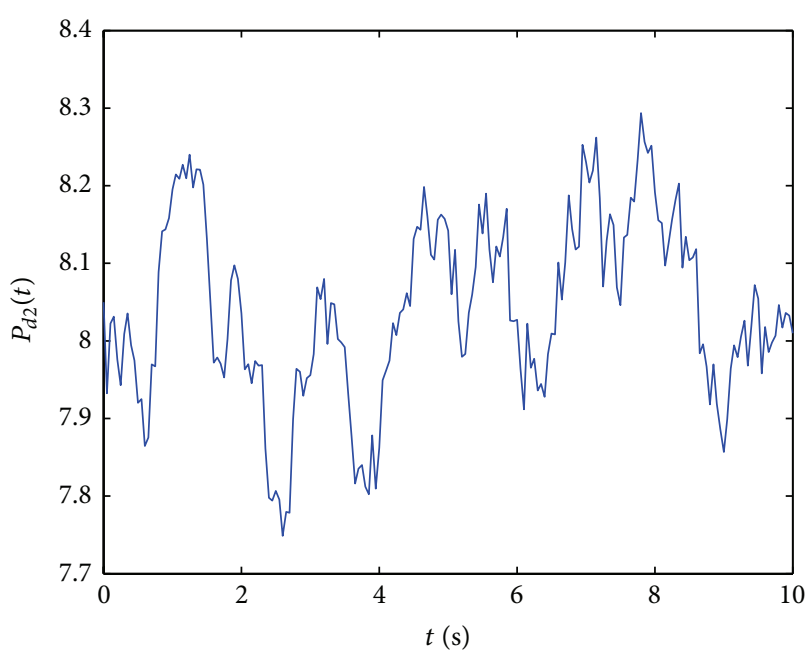

FIGURE 3: The dynamic responses of demand for consumer 2 over a path.

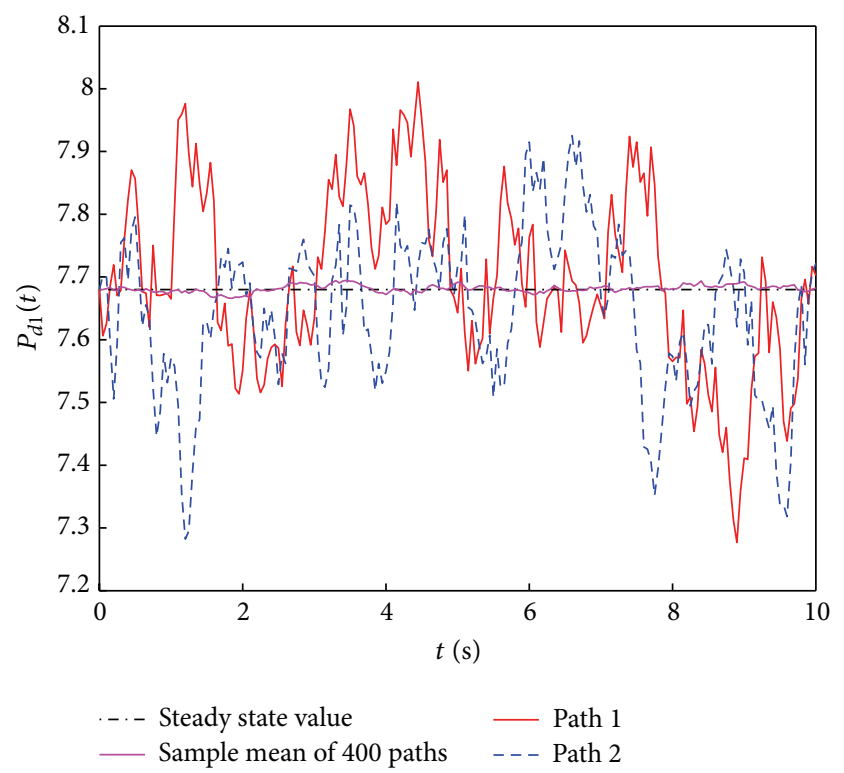

Figure 4: Average demand for consumer 1 when $Q=\left[\begin{array}{ll}0.2 & 0.3\end{array}\right]^{\mathrm{T}}$ over 400 paths.

Based on statistics theory, if one solution process is regarded as a sample from population, the average value of many solution processes is the mean value of samples. The simulation of sample mean of $P_{d 1}(t)$ and $P_{d 2}(t)$ solution processes on 400 paths is shown in Figures 4 and 5; path 1 and path 2 are two of the 400 paths. It is obvious to conclude that the sample mean variation range of the two solution processes is small and close to the steady state values 7.68 and 8.05, respectively. It is implied that $P_{d 1}(t)$ and $P_{d 2}(t)$ are of mean stability.

Example 9. For the electricity market stochastic model in Example 8, if the demand elasticity of some suppliers became zero and other data were unchanged, for instance, we take 


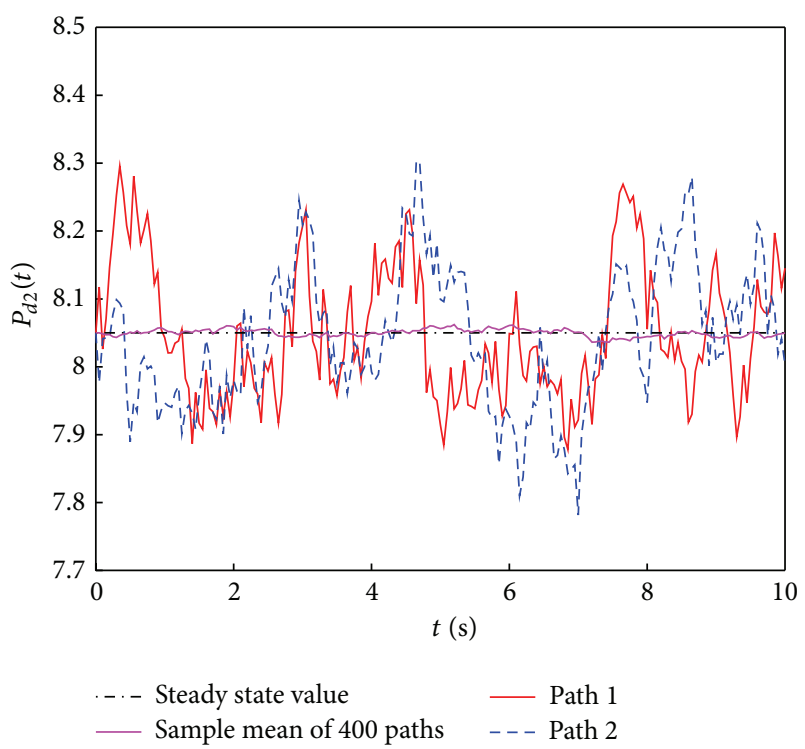

FIGURE 5: Average demand for consumer 2 when $Q=\left[\begin{array}{ll}0.2 & 0.3\end{array}\right]^{\mathrm{T}}$ over 400 paths.

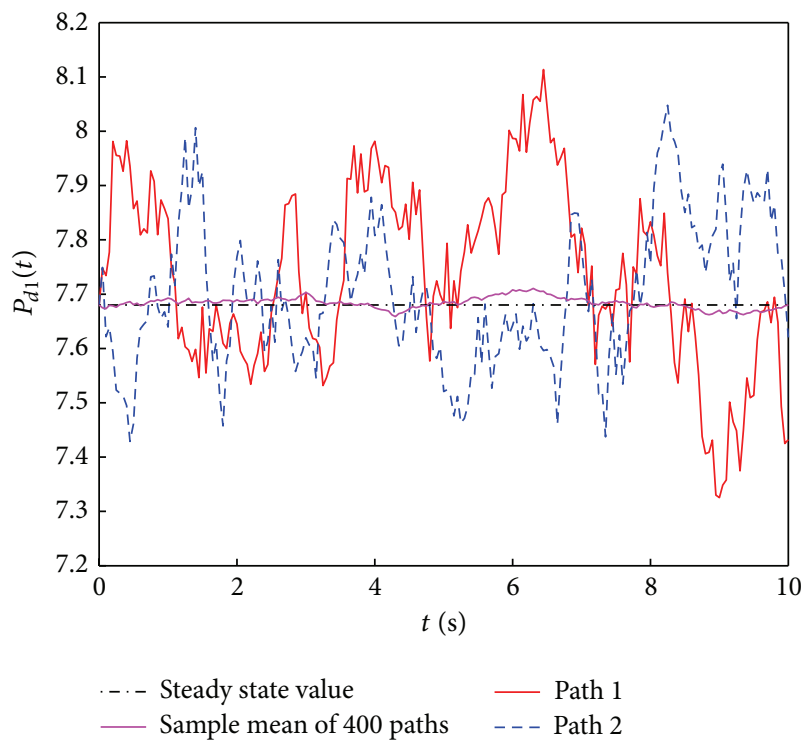

FIgURE 6: Average demand for consumer 1 when $Q=\left[\begin{array}{ll}0.2 & 0.3\end{array}\right]^{\mathrm{T}}$ over 400 paths.

$C_{1}=\operatorname{diag}\{0,0,-0.2\}$ and other data as shown in Example 8, then the diagonal elements of $C_{1}$ are nonpositive and the diagonal elements of $C_{2}$ are negative. By the corollary, this modified stochastic electricity market model is also of mean stability.

Combined with the data above, some numerical simulation and computation results are illustrated with the random excitation intensity of $Q=\left[\begin{array}{ll}0.2 & 0.3\end{array}\right]^{\mathrm{T}}$. Similar to Figures 2 and 3, the dynamic responses of $P_{d 1}(t)$ and $P_{d 2}(t)$ solution processes on a path are no longer illustrated. Figures 6 and 7 correspond to Figures 4 and 5. Graphical presentation further indicates that if the demand elasticity of suppliers

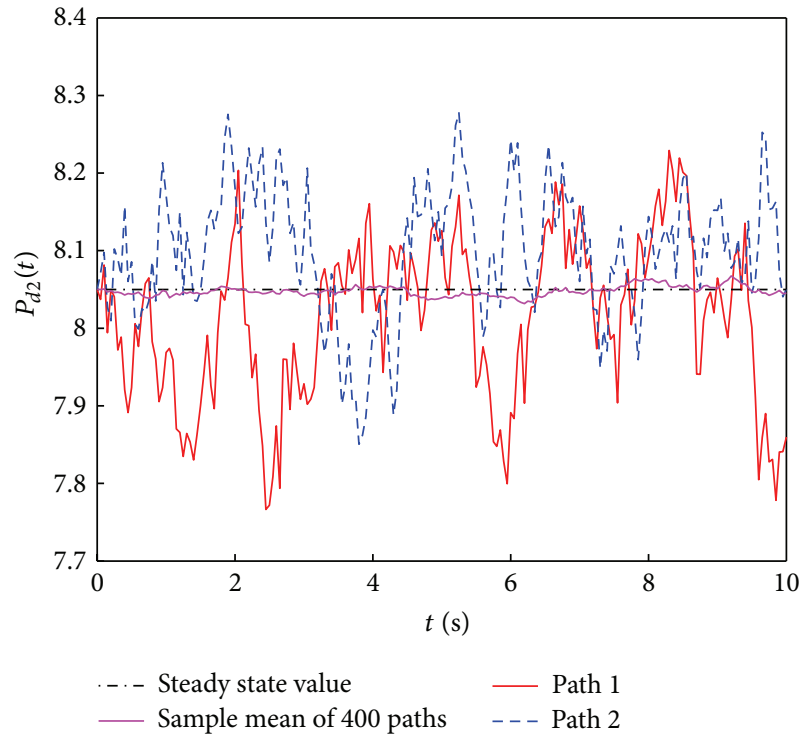

Figure 7: Average demand for consumer 2 when $Q=\left[\begin{array}{ll}0.2 & 0.3\end{array}\right]^{\mathrm{T}}$ over 400 paths.

is nonnegative and the demand elasticity of consumers is negative, then the system is of mean stability.

\section{Conclusions}

(1) Taking advantage of small Gauss type random excitation to describe the random nature of demand sides, a stochastic dynamic electricity market model is presented to reveal the dynamic characteristics of electricity market more accurately based on the deterministic electricity market dynamic model proposed by Alvarado.

(2) Using the theory of stochastic differential equations, stochastic process theory, and eigenvalue techniques, the determining conditions of the mean stability for this model are provided. The conditions manifest that the stability of electricity market can be judged directly by the initial data's symbol of the demand elasticity.

(3) For the stochastic electricity market model, numerical examples in which the data partially comes from a deterministic electricity market are analyzed and examined from a statistical viewpoint. The results of numerical examples are consistent with the stability analysis using determining conditions. It is illustrated that the determining conditions of stability are effective, practical, and advantageous.

Based on the deterministic electricity market dynamic model proposed by Alvarado, a stochastic electricity market model is provided and its stability is studied. In recent years, the increase of the proportion of random power capacity such as renewable energy sources will bring great influence on the stability of the power market. The effect of randomness as a result of renewable energy sources on electricity market will be researched in the next step. 


\section{Conflict of Interests}

The authors declare that there is no conflict of interests regarding the publication of this paper.

\section{Acknowledgment}

This work was supported in part by the National Natural Science Foundation of China, Grant no. 51190103.

\section{References}

[1] B. Beavis and I. M. Dobbs, Optimisation and Stability Theory for Economic Analysis, Cambridge University Press, Cambridge, UK, 1990.

[2] F. Alvarado, "The stability of power system markets," IEEE Transactions on Power Systems, vol. 14, no. 2, pp. 505-511, 1999.

[3] F. L. Alvarado, J. Meng, W. S. Mota, and C. L. DeMarco, "Dynamic coupling between power markets and power systems," in Proceedings of the IEEE Power Engineering Society Summer Meeting, pp. 2201-2205, Seattle, Wash, USA, July 2000.

[4] F. L. Alvarado and W. S. Mota, "The role of energy imbalance management on power market stability," in Proceedings of the 31st Annual Hawaii International Conference on System Sciences (HICSS '98), pp. 4-8, January 1998.

[5] W. S. Mota and F. L. Alvarado, "Power markets stability considering energy imbalance," in Proceedings of the IEEE International Symposium on Circuits and Systems (ISCAS '00), pp. 665-668, Geneva, Switzerland, May 2000.

[6] D. H. M. Nguyen and K. P. Wong, "A general stability metric for competitive power markets," in Proceedings of the International Conference on Electric Utility Deregulation and Restructuring and Power Technologies (DRPT '00), 2000.

[7] Y. S. Xue, "Interactions between power market stability and power system stability," Automation of Electric Power Systems, vol. 26, no. 21, pp. 1-6, 2002.

[8] Y. S. Xue, "Interactions between power market stability and power system stability," Automation of Electric Power Systems, vol. 26, no. 21-22, pp. 1-4, 2002.

[9] J. Shang, Y. Huang, and Q. Xia, Research on Electricity Market Theory and Its Applications, Electric Power Press, Beijing, China, 2002.

[10] Z.-F. Tan, G.-Q. Tang, E.-K. Yu, and Y. Li, "Power market dynamic analysis," Proceedings of the Chinese Society of Electrical Engineering, vol. 21, no. 12, pp. 88-92, 2001.

[11] Y.-H. Hou and F. F. Wu, "Stability of electricity market with cyclic load demand," Proceedings of the Chinese Society of Electrical Engineering, vol. 26, no. 24, pp. 12-17, 2006.

[12] J. Nutaro and V. Protopopescu, "The impact of market clearing time and price signal delay on the stability of electric power markets," IEEE Transactions on Power Systems, vol. 24, no. 3, pp. 1337-1345, 2009.

[13] D. Watts and F. L. Alvarado, "The influence of futures markets on real time price stabilization in electricity markets," in Proceedings of the 37th Hawaii International Conference on System Sciences (HICSS '03), 2003.

[14] S. K. M. Kodsi, Accounting for the Effects of Power System Controllers and Stability on Power Dispatch and Electricity Market Prices, University of Waterloo, Waterloo, Canada, 2005.
[15] Z. H. Yang, Y. F. Liu, Y. Tang, and F. L. Wu, "Analysis of power market stability," Proceedings of the Chinese Society for Electrical Engineering, vol. 25, no. 2, pp. 1-5, 2005.

[16] Z. H. Lu, G. Y. Li, and M. Zhou, "Study of electricity market stability model," in Proceedings of the 7th IET International Conference on Advances in Power System Control, Operation and Management (APSCOM '06), p. 369, Hong Kong, 2006.

[17] S. V. Dhople, Y. C. Chen, L. Deville, and A. D. DominguezGarcia, "Analysis of power system dynamics subject to stochastic power injections," IEEE Transactions on Circuits and Systems I: Regular Papers, vol. 60, no. 12, pp. 3341-3353, 2013.

[18] P. Parinya, A. Sangswang, K. Kirtikara, D. Chenvidhya, S. Naetiladdanon, and C. Limsakul, "A study of effects of stochastic wind power and load to power system stability using stochastic stability analysis method," Advanced Materials Research, vol. 931-932, pp. 878-882, 2014.

[19] T. Odun-Ayo and M. L. Crow, "Structure-preserved power system transient stability using stochastic energy functions," IEEE Transactions on Power Systems, vol. 27, no. 3, pp. 14501458, 2012.

[20] J. Zhang, P. Ju, Y. Yu, and F. Wu, "Responses and stability of power system under small Gauss type random excitation," Science China Technological Sciences, vol. 55, no. 7, pp. 18731880, 2012.

[21] B. G. Zhang and Y. Z. Zhao, Stochastic Differential Equations in Science and Engineering, Ocean Press, Beijing, China, 1998.

[22] K. Benjelloun and E. K. Boukas, "Mean square stochastic stability of linear time-delay system with Markovian jumping parameters," IEEE Transactions on Automatic Control, vol. 43, no. 10, pp. 1456-1460, 1998.

[23] H. M. Ahmed, "Euler-Maruyama numerical solution of some stochastic functional differential equations," Journal of the Korean Society for Industrial and Applied Mathematics, vol. 11, no. 1, pp. 13-30, 2007.

[24] S. G. Hu, C. M. Huang, and F. K. Wu, Stochastic Differential Equations, Science, Beijing, China, 2008. 


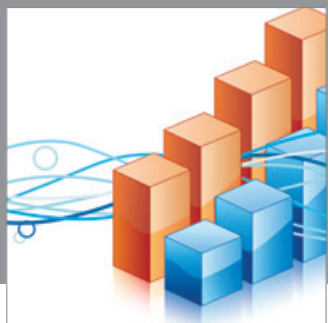

Advances in

Operations Research

mansans

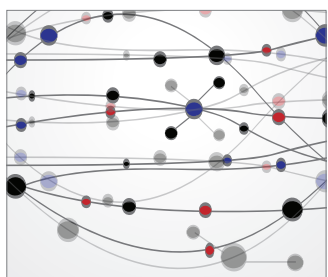

The Scientific World Journal
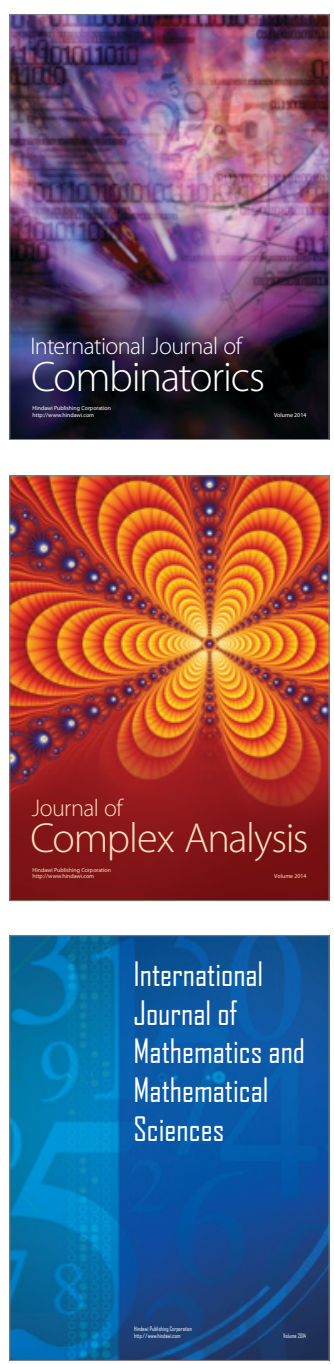
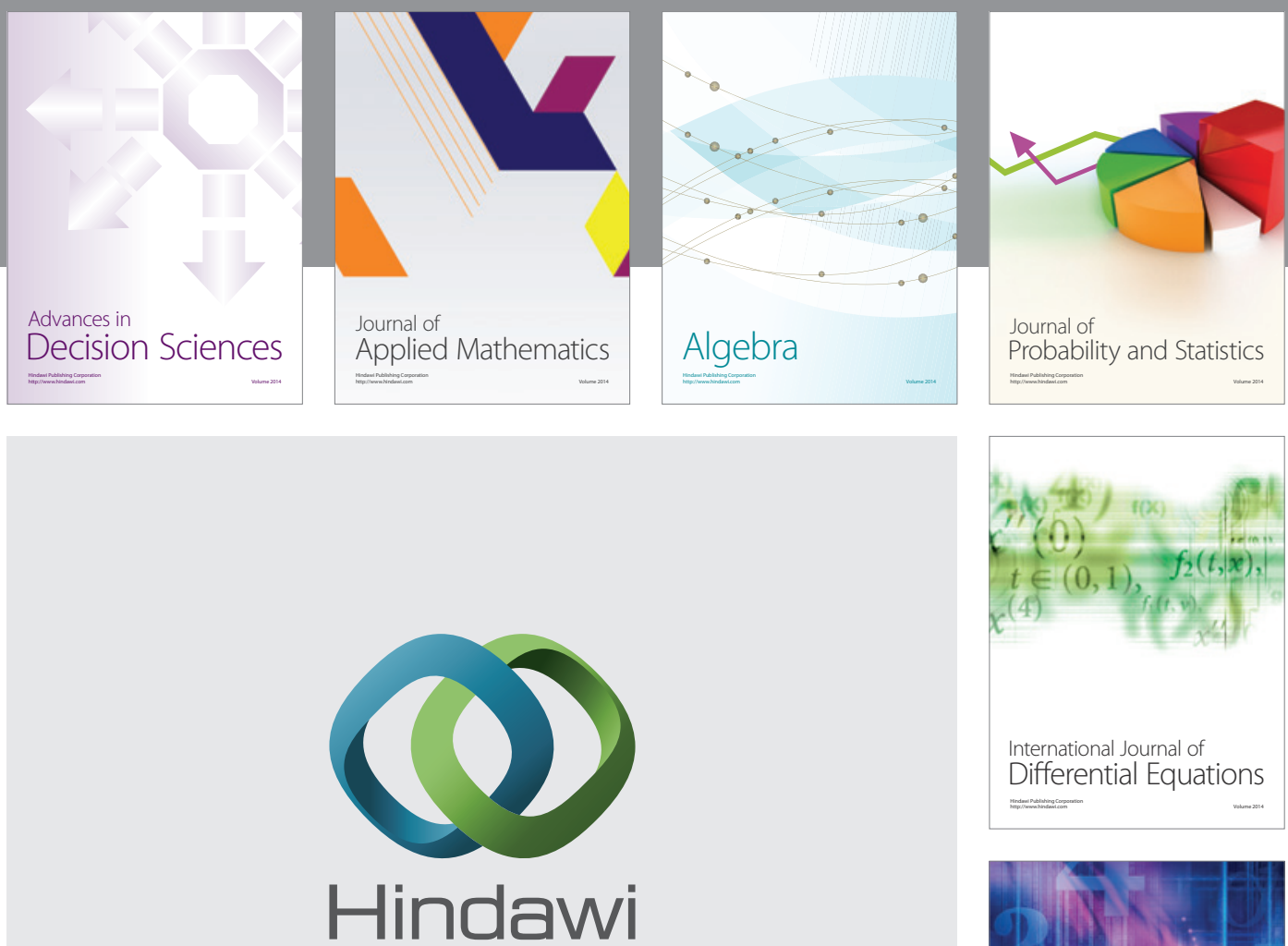

Submit your manuscripts at http://www.hindawi.com
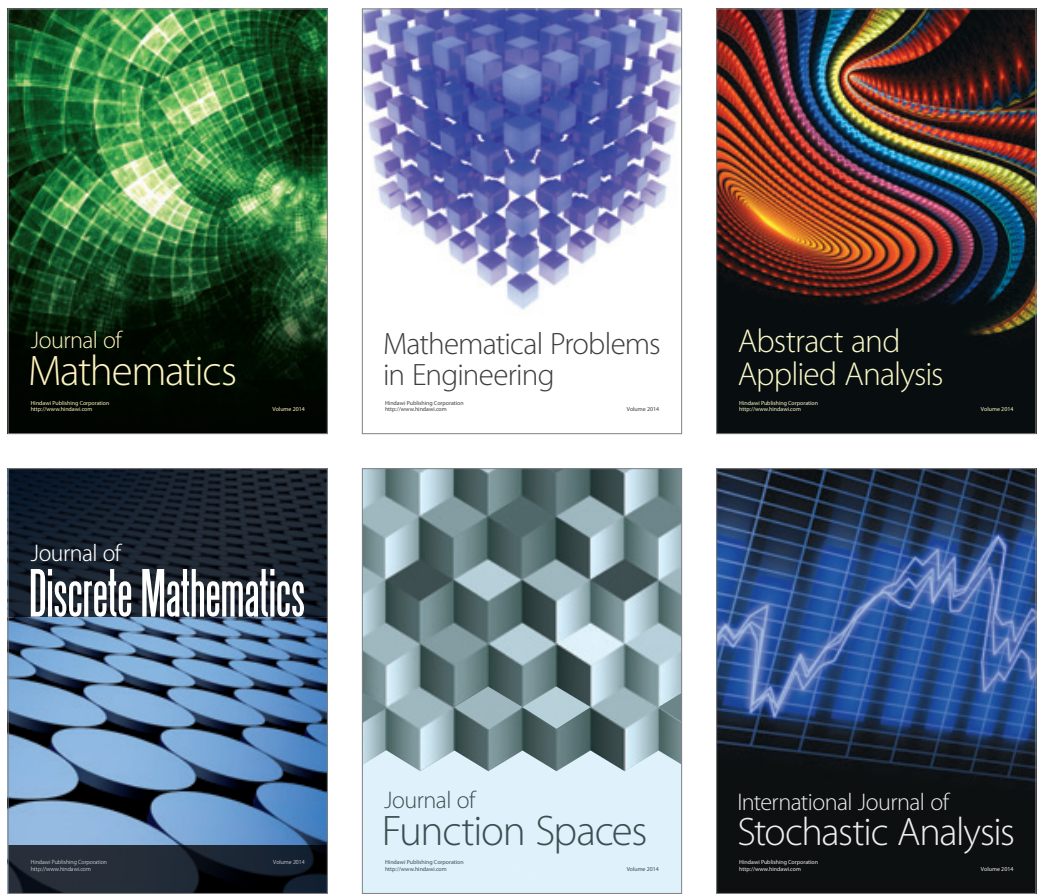

Journal of

Function Spaces

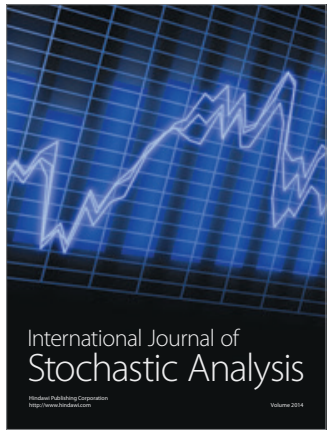

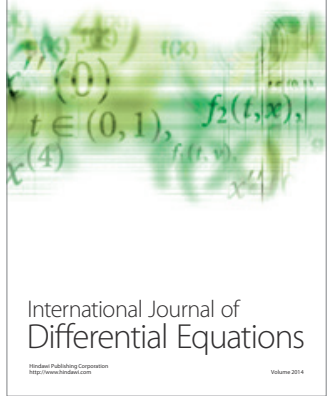
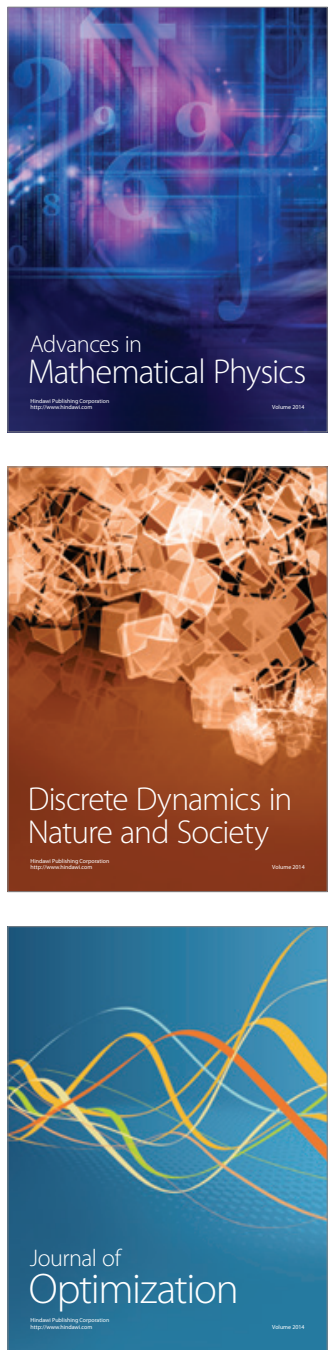